\title{
Individual and family environment correlates differ for consumption of core and non-core foods in children
}

\author{
Laura Johnson, Cornelia H. M. van Jaarsveld and Jane Wardle* \\ Cancer Research UK Health Behaviour Research Centre, Department of Epidemiology and Public Health, \\ University College London, London WC1E 6BT, UK
}

(Received 9 March 2010 - Revised 9 August 2010 - Accepted 4 October 2010 - First published online 29 November 2010)

\begin{abstract}
Children's diets contain too few fruits and vegetables and too many foods high in saturated fat. Food intake is affected by multiple individual and family factors, which may differ for core foods (that are important to a healthy diet) and non-core foods (that are eaten more for pleasure than health). Data came from a sample of twins aged 11 years ( $n$ 342) and their parents from the Twins Early Development Study. Foods were categorised into two types: core (e.g. cereals, vegetables and dairy) and non-core (e.g. fats, crisps and biscuits). Parents' and children's intake was assessed by an FFQ. Mothers' and children's preference ratings and home availability were assessed for each food type. Parental feeding practices were assessed with the child feeding questionnaire and child television (TV) watching was maternally reported. Physical activity was measured using accelerometers. Correlates of the child's consumption of each food type were examined using a complex samples general linear model adjusted for potential confounders. Children's non-core food intake was associated with more TV watching, higher availability and greater maternal intake of non-core foods. Children's core food intake was associated with higher preferences for core foods and greater maternal intake of core foods. These results suggest that maternal intake influences both food types, while preferences affect intake of core foods but not of non-core foods, and availability and TV exposure were only important for non-core food intake. Cross-sectional studies cannot determine causality, but the present results suggest that different approaches may be needed to change the balance of core and non-core foods in children's diets.
\end{abstract}

Key words: Core food: Non-core food: Children: Diet

A balanced diet with adequate intakes of at least thirty-four nutrients in childhood is essential for optimal growth and development, school performance, behaviour and prevention of diseases such as asthma or obesity ${ }^{(1-4)}$. Results from the Health Survey for England ${ }^{(5)}$ and the National Diet and Nutrition Survey ${ }^{(6)}$ indicate that $80 \%$ of English children do not meet the target of eating five portions of fruits and vegetables a day, and saturated fat intakes were nearly $50 \%$ higher than the recommended level. Change is urgently required to improve the diets of children to encourage healthy growth and development and prevent the development of chronic disease in adult life.

Dietary quality is a multidimensional concept characterised in many different ways. High fat, low vitamins, large portions, excess sugar, low fibre, insufficient $\mathrm{Fe}$, and too few whole grains, fruits or vegetables all could indicate an unhealthy diet. Nutrient intakes have traditionally been used to reflect diet quality, but there is an increasing realisation that practical dietary advice needs to be based on foods rather than nutrients, because in reality this is what people eat ${ }^{(7)}$. Defining single foods as good or bad can be problematic, as their effects can vary depending on the health outcome; for instance, breakfast cereals fortified with vitamins and minerals can benefit growth but if also high in sugar this can harm dental health. One alternative approach is to categorise foods as essential (core) or superfluous (non-core) to a healthy diet, a method that has been used in the study of children's diet ${ }^{(8-11)}$. The Australian dietary guidelines are based on five core food groups (1, carbohydrate-rich; 2, vegetables; 3 , fruits; 4, dairy products; 5, high-protein) with all other foods defined as extra or noncore foods $^{(8)}$. Non-core foods are more energy dense than core foods and lower in nutrients required for health ${ }^{(10,11)}$ and should ideally be eaten in moderation. Although it could be argued that non-core foods can provide some useful nutrition, for example essential fat intake, a diet based on core food adequately meets essential requirements and thus any nutrition from non-core foods can be viewed as extra. Characterising intakes in this way can provide a simple index of diet quality for use in large-scale research.

Children's intakes are reflection of the foods that are familiar and preferred. Foods that are available, accessible,

Abbreviations: TEDS, Twins Early Development Study; TV, television. 
advertised on television (TV) or eaten by parents or peers will tend to be eaten more frequently ${ }^{(12)}$. Knowing the correlates of food intake in children can help to inform the development of effective interventions to achieve positive dietary change. Most attention has been paid to fruit and vegetable consumption, with fewer studies investigating less healthy foods such as fast food or high-sugar snacks and drinks ${ }^{(13,14)}$. It is possible that different factors influence the intake of core foods that are part of a healthy diet and non-core foods that are eaten more for pleasure. Preferences are a strong driver of food intake because children will often reject foods they do not like ${ }^{(12)}$. A recent review found a positive association between preferences and intake of fruits and vegetables in eleven of thirteen studies ${ }^{(14)}$. Similar evidence for non-core foods is incomplete, but suggests that the impact of preferences may be limited ${ }^{(14)}$. TV watching is often related to consumption of unhealthy foods even after controlling for a range of family environment factors ${ }^{(15-17)}$. Exposure to adverts, which are usually biased towards non-core food varieties, has been associated with more requests, higher preferences and greater intake of these foods in children ${ }^{(18-20)}$. Higher levels of physical activity have also been related to a healthier dietary intake in adults ${ }^{(21-23)}$. Few studies have investigated the relationship in children, and results have been weak or inconsistent ${ }^{(24,25)}$. For example, lower fruit and vegetable intake was associated with less activity in girls but not in boys, and more snacks were associated with higher activity in younger children but not in older children ${ }^{(24)}$.

In addition to individual factors such as preferences, the family environment can have a powerful effect in shaping children's food intake. Children cannot eat foods that are not available to them, and given that they eat at least two-thirds of their meals at home, the range of foods available in the home sets limits to their possible food choices $^{(19)}$. One study found that fruit, but not vegetable, availability was correlated with intake, while availabilities of savoury and sweet snacks were associated with higher intake of non-core foods ${ }^{(9)}$. However, two reviews have reported that overall evidence is inconsistent both for the core and for the limited range of non-core foods investigated $^{(13,14)}$. Parents are an important element of the family environment. They buy food for the home and are responsible for children's access to food through their feeding practices. Practices such as 'restriction' and 'pressure to eat' typically aim to lower intake of 'junk' foods or increase intake of core foods, but experimental evidence suggests that they can be counter-productive, with higher 'junk' food consumption often observed ${ }^{(26)}$. However, associations between parental feeding styles and children's diet could also reflect parents reacting to their children's refusals to eat core foods or requests for non-core foods, suggesting that diet is a cause not a consequence of parental feeding practices ${ }^{(26)}$. Finally, parents' own intakes serve as a model of eating for their children to learn from and are one of the most commonly documented correlates of food intake in children ${ }^{(13)}$. One might expect parental food preferences to serve as a model in a similar way; however, evidence suggests a weak relationship between parents' and children's food preferences, but this may change with age as children are exposed to a wider range of foods because exposure is a powerful determinant of preferences ${ }^{(27-29)}$.

The multiple determinants of children's food intake are inevitably interlinked, e.g. TV watching may influence children's preferences and home availability if requests for non-core foods increase. Parents' food intake and preferences are also likely to be reflected in availability. Most existing investigations look at univariable relationships neglecting the potential inter-correlations ${ }^{(14,30)}$. A multivariable approach could identify the key players in the development of food intake in children. Therefore, the aim of this analysis was to take a multidimensional approach in assessing the independent correlates of core and non-core food intake in children.

\section{Methods}

Sample

Data came from questionnaires collected in a subsample of twins in the Twins Early Development Study (TEDS). The TEDS is a population-based sample of over 15000 twin pairs born in the UK between 1994 and 1996(31). The families described in the present study were taking part in an investigation of genetic and environmental influences on appetite and growth, and had been selected so that half had two overweight or obese parents (BMI $>27 \mathrm{~kg} / \mathrm{m}^{2}$ ) and half had lean parents (BMI $<25 \mathrm{~kg} / \mathrm{m}^{2}$ ). The groups were matched for geography and paternal occupation ${ }^{(32)}$. Both groups contributed data to the present study, with results combined after testing for parental weight group differences in children's food intake to confirm that the two samples were homogeneous.

At baseline in 1999, the sample consisted of 428 children from 214 families (100 overweight or obese and 114 normalweight families), from all over England and Wales. Families were contacted again in 2006 when children were aged 11 years, and data from 346 children (173 families) were obtained; a follow-up rate of $80 \%$. At both times, families were visited at home where children completed behavioural tasks and mothers completed questionnaires. The present study was conducted according to the guidelines laid down in the Declaration of Helsinki, and all procedures involving human subjects were approved by the University College London Committee for the Ethics of non-National Health Service Human Research. Verbal informed consent was obtained from all parents on the telephone before the home visit, and written consent forms were completed by parents on behalf of themselves and their children at the start of the home visit. 


\section{Classification of foods as core or non-core}

Each food group in the FFQ, food availability or food preference questionnaires was defined as a core or a non-core food (see Table 1 for examples) based on the Australian dietary guidelines ${ }^{(8)}$. Core foods were defined as those included in the five core groups: 1 - bread, cereals, rice, pasta and noodles; 2 - vegetables; 3 - fruits; 4 - dairy products; 5 - meat and fish. All other foods were classified as non-core. For most foods, it was obvious when to classify them as core and non-core. When it was not obvious, previous research was consulted ${ }^{(8-11)}$. For example, fruit juice was considered a core food, but fruit squash was defined as non-core and meat was classified as non-core if it was processed. A complete list of core and non-core foods can be requested from the authors.

\section{Food intake}

Children's food intake was assessed at age 11 (SD 0.5) years using a validated FFQ completed by the mother ${ }^{(33)}$. Mothers reported their child's frequency of consumption of forty-five foods or food groups with response options of 'never', 'once a month', 'once a fortnight', 'once a week', '2 d a week', '3d a week', '4d a week', '5d a week', ' $6 \mathrm{~d}$ a week' or 'every day'. Data were recoded to provide values reflecting frequencies per day: for example, 'every day' was recoded as 1 (time/d), 'once a week' was recoded as $1 / 7$ (times/d), once a fortnight was recoded as $1 / 14$ (times/d), etc. Total daily consumption frequency (times/d) of core (twenty-seven foods) and non-core foods (eighteen foods) by the child was calculated by summing the daily intakes. Parents' self-reported food intake

Table 1. Examples of foods defined as core and non-core

\begin{tabular}{ll}
\hline Core foods & Non-core foods \\
\hline Bread (all types) & Sweet biscuits \\
Beans & Cakes and scones \\
Chicken (turkey and duck) & Sweets and chocolate \\
Lamb (chops, roast and stew) & Sugar-sweetened drinks \\
& and fruit squash/cordial \\
Liver or kidney & Artificially sweetened drinks \\
Beef & Soft drinks (all types) \\
Cheddar or other cheese & Bacon, ham and pork \\
Cottage cheese & Processed meats \\
Eggs & Sausages \\
Fish not in batter or crumb & Fish in batter or crumb \\
Oily fish & Chips, fried or roast potatoes \\
Fruit & Crisps \\
Green cooked vegetables & Butter \\
Other cooked vegetables & Margarine (all types) \\
Salad (including raw vegetables) & Other oils and fats \\
Yams, sweet potatoes and plantains & \\
Fruit juices (with no sugar added) & \\
Potatoes (boiled, mashed and baked) & \\
Pasta & \\
Rice & \\
Breakfast cereals (all types) & \\
Milk (all types) & \\
Yogurt & \\
\hline & \\
\hline
\end{tabular}

was assessed in 1999 using a FFQ validated for adults ${ }^{(34)}$, and total daily consumption frequency (times/d) of core (seventy-three foods) and non-core foods (fifty-seven foods) was calculated by summing intakes of core and non-core foods, respectively.

\section{Food preferences and availability}

Child and maternal food preferences were assessed at follow-up by food preference questionnaires used in the baseline assessment, which were developed using food lists in the adult and child $\mathrm{FFQ}^{(35)}$. Children reported their own food preferences, an approach that has been used previously ${ }^{(36)}$. Foods were presented as a written list and were rated on a five-point scale from hate it $=1$ to love it $=5$, a separate response indicated if any foods had not been tried. Average preference ratings for all core (child = seventy-four foods; mother = ninety foods) and non-core (child = thirty-three foods; mother $=$ thirtysix foods) foods that had been tried were calculated separately. Home food availability was measured by a questionnaire at follow-up by the mother reporting whether a list of 118 foods were currently present or absent from the home, and total number of core (out of eighty-three foods) and non-core (out of thirty-five foods) foods available in the home was calculated.

\section{Parental feeding practices}

Parental feeding practices were measured at follow-up using validated scales from the child feeding questionnaire $^{(37)}$ and the parental feeding style questionnaire ${ }^{(38)}$ completed by the mother. Monitoring (three items), restriction (eight items), pressure to eat (four items) and encouragement (eight items) scales were created by taking the average of responses to items on a Likert scale of either 1 (never) to 5 (always) or 1 (disagree) to 5 (agree).

\section{Television watching and physical activity}

TV watching by the child during the week and at the weekend was reported by the mother, and total TV watching (h/week) was computed. Activity was measured using the Actigraph model 7164 accelerometer, which is the most valid and commonly used device in children ${ }^{(39)}$. Actigraph data files were processed using the MAHUffe program (www.mrc-epid.cam. ac.uk/Research/PA/downloads.html), and total physical activity (mean accelerometer counts per minute) was calculated.

\section{Data analysis}

Data are presented as means and standard deviations or percentages. Differences in preferences for core and non-core foods were examined using paired $t$ tests. Analyses of the correlates of core and non-core food intake in children used a complex samples general linear model 
to take account of the clustering of twins within families. Each potential correlate was entered into a basic model with either child's core or child's non-core food intake as the outcome. All significant $(P<0.05)$ correlates identified from the basic models were individually entered into an adjusted model containing child's age, sex, and BMI standard deviation score, maternal education and family weight group as covariates with either child's core or child's non-core food intake as the outcome. In fully adjusted models, all significant correlates identified in the basic models were added to the adjusted model to identify independent correlates of either core or non-core food intake in children.

Data were missing for varying numbers of participants on different potential correlates. For univariate analyses, all available data were used to look at relationships between core or non-core food intake and individual variables. In the fully adjusted model, only cases with available data on all variables were included in the analysis ( $n$ 223). The reduced sample size was largely a result of missing data on maternal food intake; therefore, we also imputed maternal food intake data by replacing missing values with the sample mean. Analyses were repeated using the imputed data to establish whether a larger sample size modified the estimates and significance of all other covariates included in the fully adjusted models.

The effects of potential correlates of core and non-core food intake were directly compared in two further models with core or non-core food intake as the outcomes. Both models contained all significant $(P<0.05)$ correlates identified from the fully adjusted models for both core and non-core foods as well as child's age, sex, and BMI standard deviation score, maternal education and family weight group as covariates. Maternal food preferences were additionally included because results using the larger sample with imputed data on maternal food intake suggested that maternal food preference was a significant correlate of children's core food intake. All variables were standardised by calculating $z$-scores $(z$-score $=$ (individual value - mean)/standard deviation) before entering the models, and model effect estimates from the core $\left(b_{1}\right.$ and $\left.\mathrm{se}_{1}\right)$ and non-core food intake $\left(b_{2}\right.$ and $\left.\mathrm{se}_{2}\right)$ models were compared using a $Z$ test $\left(Z=\left(b_{1}-b_{2}\right)\right.$ / $\left(\mathrm{se}_{1}^{2}+\mathrm{se}_{2}^{2}\right.$ ), exact $P$ values are obtained from standard normal distribution tables) ${ }^{(40,41)}$. Analyses were completed in SPSS version 15 (SPSS, Inc., Chicago, IL, USA).

\section{Results}

The presence of significant differences in food intake between children from families with overweight and lean parents was examined. Mean consumption of core foods (lean: 8.0 (SD 0.2); overweight: 8.2 (SD 0.2)) or non-core foods (lean: $5 \cdot 0$ (SD $0 \cdot 1)$ ); overweight: $5 \cdot 2$ (SD $0 \cdot 2)$ ) did not vary by parental weight group; therefore, the groups were combined. Descriptive characteristics of individual and family environment factors are displayed in Table 2. Children's preferences for non-core foods were significantly higher than their preference for core foods $(P<0 \cdot 0001)$.

\section{Correlates of core food intake}

Basic models indicated that a higher intake of core foods by the child was associated with a higher preference for core foods by the child and the mother, a greater availability of core foods in the home, a higher intake of core foods by the mother and a more encouraging parental feeding style (Table 3). There was no evidence of an association between child's core food intake and time spent watching TV, physical activity, other parental feeding styles or paternal core food intake. After adjusting for child's sex, age, and BMI standard deviation score, maternal education and parental weight group, estimates remained similar for the effect of food preferences, maternal core food intake and an encouraging feeding style (Table 3). The estimate for the effect of availability of core foods on child's core food consumption was reduced slightly from 0.05 to 0.03 and no longer reached statistical significance. In the fully adjusted model ( $n$ 223) that included all correlates identified in the basic models, only the child's preference for core foods and maternal intake of core foods showed evidence of an independent association with the core food intake (Table 3). The effect estimate of maternal core food preferences on child's core food intake was reduced by half and no longer reached statistical significance. In the fully adjusted model including imputed values for cases with missing maternal core food intake data ( $n$ 301), maternal core food intake $(0 \cdot 12,95 \%$ CI $0.06,0 \cdot 18 ; P=0.0002)$, the child's core food preferences (0.75, 95\% CI 0.39, 1.11; $P<0.0001)$ and the mother's core food preferences ( 0.89 , $95 \%$ CI $0 \cdot 14,1.64 ; P=0.02)$ were independently associated. Home food availability $(0 \cdot 00,95 \% \mathrm{CI}-0 \cdot 04,0 \cdot 03 ; P=0.88)$ and encouragement $(0.08,95 \% \mathrm{CI}-0.40,0.56 ; P=0.74)$ were attenuated and non-significant.

Inter-correlations between the correlates of child's core food intake are reported in Table 4. Maternal core food intake was moderately $(r 0 \cdot 4)$ and the child's preference for core foods was weakly $(r \quad 0 \cdot 2)$ correlated with maternal core food preferences, suggesting that either or both of these factors partly mediate the association between maternal core food preferences and child's core food intake in the fully adjusted model. Availability of core foods was moderately correlated with both maternal preferences $(r 0.3)$ and intake of core foods ( $r 0.3)$, which may explain the attenuation of the effect of availability in the fully adjusted model. Attenuation of the effect of encouragement on child's core food intake could be accounted for by the moderate correlation between encouragement and maternal preference for core foods $(r 0.35)$ and the weak correlation with core food availability $(r 0 \cdot 2)$. 
Table 2. Descriptive characteristics of individual and family environment factors (Mean values and standard deviations or percentages)

\begin{tabular}{|c|c|c|c|}
\hline & $n$ & Mean & SD \\
\hline \multicolumn{4}{|l|}{ Child characteristics } \\
\hline Sex (\% female) & 342 & \multicolumn{2}{|c|}{56} \\
\hline Age in 2006 (years) & 342 & $11 \cdot 17$ & 0.54 \\
\hline BMI SDS in $2006\left(\mathrm{~kg} / \mathrm{m}^{2}\right)$ & 335 & 0.47 & $1 \cdot 18$ \\
\hline Overweight or obese in 2006 (\%) & 335 & \multicolumn{2}{|c|}{25} \\
\hline Core food intake (times/d) in 2006 & 342 & 8.09 & 1.87 \\
\hline Non-core food intake (times/d) in 2006 & 342 & 5.06 & $1 \cdot 3$ \\
\hline Core food preference in $2006^{\star}$ & 340 & 3.57 & 0.57 \\
\hline Non-core food preference in $2006^{\star}$ & 340 & 4.01 & 0.46 \\
\hline TV watching (h/week) in 2006 & 340 & 12.57 & $6 \cdot 12$ \\
\hline Activity (counts/min) in 2006 & 249 & 687.49 & $262 \cdot 14$ \\
\hline \multicolumn{4}{|l|}{ Parent characteristics } \\
\hline Maternal age in 2006 (years) & 171 & 41.48 & $4 \cdot 27$ \\
\hline Maternal BMI in $2006\left(\mathrm{~kg} / \mathrm{m}^{2}\right)$ & 167 & 30.01 & $7 \cdot 288$ \\
\hline Maternal education in 1999 (\%) & & & \\
\hline No qualifications & & \multicolumn{2}{|c|}{5} \\
\hline GCSE & & \multicolumn{2}{|c|}{63} \\
\hline A-levels & 171 & \multicolumn{2}{|c|}{13} \\
\hline Higher national certificate or diploma & & \multirow{2}{*}{\multicolumn{2}{|c|}{$\begin{array}{c}6 \\
13\end{array}$}} \\
\hline Undergraduate or postgraduate degree & & & \\
\hline Maternal core food intake (times/d) in 1999 & 121 & 14.37 & 4.96 \\
\hline Maternal non-core food intake (times/d) in 1999 & 121 & $15 \cdot 09$ & 4.02 \\
\hline Maternal core food preference in $2006^{\star}$ & 171 & 3.8 & 0.39 \\
\hline Maternal non-core food preference in $2006^{\star}$ & 171 & 3.55 & 0.44 \\
\hline Paternal core food intake (times/d) in 1999 & 110 & $13 \cdot 19$ & $3 \cdot 10$ \\
\hline Paternal non-core food intake (times/d) in 1999 & 110 & 18.02 & $5 \cdot 35$ \\
\hline Monitoring in 2006 & 171 & 4.03 & 0.72 \\
\hline Restriction in 2006 & 171 & 3.03 & $0 \cdot 81$ \\
\hline Pressure in 2006 & 171 & $2 \cdot 27$ & 0.93 \\
\hline Encouragement in 2006 & 171 & 3.87 & 0.54 \\
\hline Core food availability (total out of eighty-four foods) in 2006 & 171 & 39.43 & $7 \cdot 88$ \\
\hline Non-core food availability (total out of thirty-five foods) in 2006 & 171 & $17 \cdot 27$ & 4.99 \\
\hline Core food availability ( $\%$ of eighty-four foods) in 2006 & 171 & 47 & 9 \\
\hline Non-core food availability (\% of thirty-five foods) in 2006 & 171 & 49 & 14 \\
\hline
\end{tabular}

SDS, standard deviation score; TV, television; GCSE, General Certificate of Secondary Education. ${ }^{\star}$ Scores range from 1 to 5 and a higher score reflects a greater preference.

\section{Correlates of non-core food intake}

Basic models indicated that a greater intake of non-core foods by children was associated with a greater availability of non-core foods in the home, a higher intake of non-core foods by the mother and more TV watching by the child (Table 3). There was no evidence of an association between non-core food intake and food preferences of either the child or the mother, child's physical activity, any of the parental feeding styles or paternal intake of non-core foods. After adjusting for child's sex, age, and BMI standard deviation score, maternal education and parental weight group, estimates of the effects identified in the basic models remained the same (Table 3 ). In the fully adjusted model, where all correlates identified in the basic models were included together as independent variables, greater intake of non-core foods by the child was independently associated with availability of non-core foods in the home, maternal intake of non-core foods and time spent watching TV by the child (Table 3).

Inter-correlations between the independent covariates of non-core food intake by the child are displayed in Table 5 . These correlations suggest that TV watching is weakly associated with maternal intake of non-core food ( $r 0 \cdot 21)$ but not with availability of non-core foods in the home $(r 0 \cdot 1)$ (Table 3).

\section{Comparing the independent correlates of core and non-core food intake}

A comparison of the standardised effects of availability, maternal and child's food preference, TV watching and maternal food intake on core and non-core food consumption is displayed in Fig. 1. Availability and TV watching were associated with a higher non-core food intake, whereas children's preferences were only associated with a higher core food intake. The effect estimates of maternal food intake and preferences did not differ significantly for core and non-core foods, with a higher maternal intake being associated with a higher intake of both food types by children. The standardised model was repeated with imputed maternal food intake data ( $n$ 297), and the results were largely unchanged. With imputed maternal intake data, the standardised estimate for maternal core food preferences with children's core food intake was significant 
(estimate 0.17 (se 0.09 ); $P=0.02$ ), but there was no significant difference when compared with the effect estimate of maternal preferences with non-core food intake (estimate 0.09 (SE 0.09), $Z$ test; $P=0.48$ ). In the final standardised models, a total of 36 and $26 \%$ of the variance was explained for core and non-core food intake, respectively.

\section{Discussion}

The present study suggests important differences in the correlates of core and non-core food consumption in children. Maternal intake of both food types was independently associated with the child's intake, but preferences were only associated with intake of core foods, while food availability and TV exposure were only associated with non-core food intake.

Maternal intake was associated with a higher consumption of both core and non-core foods in the present analysis. This confirms existing work which has repeatedly shown a positive correlation between parents' and children's intakes ${ }^{(13)}$. Interestingly, in the present study, there was no evidence of an association between paternal and child's food intake. Maternal intake has been studied more frequently, making the role of paternal intake in relation to their child's intake less clear. A study of similarity in parents' and children's nutrient intakes found weaker correlations between children and fathers than children and mothers ${ }^{(42)}$, although this differential effect of mothers and fathers is not supported consistently by other studies ${ }^{(43,44)}$. Mothers report greater perceived responsibility for feeding their child ${ }^{(45)}$, suggesting that fathers play a more limited role in children's diet. There were also more missing data for paternal intakes in the present study, so if the effect on child's intake is weaker than that of maternal intakes, then a larger sample may be required to detect the effect.

In the present analyses, children's food preferences were related to the intake of core foods but not of non-core foods. This different effect may be explained by the variation in overall preference ratings for each food type; non-core foods tended to be liked more than core foods in the present study (as shown in Table 2), a finding that is supported by the wider literature ${ }^{(46,47)}$. For well-liked foods, other factors that limit the amount of food eaten such as availability may become more important, whereas for less palatable foods, dislike is a salient feature. The effect of maternal preferences was partially, but not fully, attenuated by the inclusion of other significant correlates of core food intake and was only marginally non-significant in the model without imputed maternal intake data. Confirmation of this effect in a larger independent sample would help to substantiate this finding.

An association was found in the present study between the availability and intake of non-core foods, which was not observed for core food intake. Previous studies have also found an association between the availability 
Table 4. Inter-correlations between significant potential correlates of core food intake (Pearson's $r$ correlation coefficients $\dagger$ )

\begin{tabular}{|c|c|c|c|c|c|}
\hline Correlations & $\begin{array}{l}\text { Child's core food } \\
\text { preference in } 2006\end{array}$ & $\begin{array}{l}\text { Maternal core food } \\
\text { preference in } 2006\end{array}$ & $\begin{array}{c}\text { Maternal core } \\
\text { food intake in } 1999\end{array}$ & $\begin{array}{l}\text { Home core food } \\
\text { availability in } 2006\end{array}$ & $\begin{array}{l}\text { Encouragement } \\
\text { in } 2006\end{array}$ \\
\hline Child's core food intake in 2006 & $0 \cdot 25^{\star}$ & $0.37^{\star \star *}$ & $0.50^{* \star *}$ & $0.21^{\star * *}$ & $0.17^{*}$ \\
\hline Child's core food preference in 2006 & & $0.20^{*}$ & 0.03 & 0.02 & 0.04 \\
\hline Maternal core food preference in 2006 & & & $0.40^{\star \star \star}$ & $0.28^{\star \star \star}$ & $0.35^{\star \star \star}$ \\
\hline Maternal core food intake in 1999 & & & & $0.32^{\star}$ & 0.16 \\
\hline Home core food availability in 2006 & & & & & $0 \cdot 20^{\star}$ \\
\hline
\end{tabular}

${ }^{\star} P<0.05,{ }^{\star \star *} P<0.0001$.

$\dagger$ Calculated by square rooting $r^{2}$ derived from a complex samples general linear model of one variable against another variable.

of non-core foods such as sweet and savoury snacks and consumption of non-core foods by children ${ }^{(9,15)}$, whereas support for a link between the availability and intake of core foods such as fruits and vegetables is inconsistent ${ }^{(13)}$. The attenuation of the effect of availability on core food intake after the inclusion of maternal food intake and child's food preferences suggests that the association with availability is coincidental with high maternal intake and child's preferences. Perhaps in the case of core foods, although some availability is clearly necessary to allow intake, a greater availability on its own is not sufficient to increase children's intakes.

TV watching has been hypothesised to increase the availability of non-core foods in the home, as these foods are advertised more frequently, which encourages children to request them more often ${ }^{(20)}$. However, in the present study, there was no correlation between TV watching and availability of non-core foods; in fact, both were independently related to children's intake. Furthermore, availability of non-core foods was correlated with maternal non-core food intake, which suggests that children's requests for foods they see on TV may have a weak influence on parent purchasing behaviour relative to the impact of the parent's own intake and preferences ${ }^{(19)}$.

Differences observed in the present study of the effect of availability and preferences on core and non-core food intake may reflect differences in the relative influence of genes and environments on preferences for these types of foods. Previous analyses of the TEDS subsample of genetic and environmental effects on child's food preferences found that genes explained just $20 \%$ of the variation in preferences for the dessert group of foods (which were primarily non-core foods) compared with 51-78\% of the variation in preferences for foods in the core group such as fruits, meat and fish ${ }^{(46)}$. Based on these previous findings, it may therefore be expected that variation in environmental factors such as availability and TV exposure affects intake of non-core foods, whereas differences in individual preferences, which may be genetically determined, explain more of the intake of core foods. However, this does not mean that environmental changes cannot affect preferences for core foods; in fact, interventions to increase exposure to vegetables by repeated tasting have been successful in improving children's liking of vegetables ${ }^{(29)}$, but rather that environmental variation that exists in the present sample (e.g. in availability) is not sufficient on its own to create differences in core food preferences or intake. Future work might substantiate this proposal by examining the heritability of core food intake and assessing the extent to which genetically based differences in core food preferences and intake are shared.

Parents should be aware of the multiple factors that can influence their child's food intake, including the impact of their own food choices and eating behaviour ${ }^{(27)}$. A parent's own commitment to eat healthily, if lacking, could undermine attempts to ensure healthful eating in their children ${ }^{(19)}$. The old adage 'Do as I say and not as I do' probably does not work when trying to convince children to eat healthily. Parents should try to provide a healthy range of foods (plenty of core foods) while limiting availability of non-core foods as well as setting a good example by eating a healthy balance of core and noncore foods themselves.

The present results must be interpreted within the context of the strengths and limitations of the study. The sample of twins and recruitment of families based on parental weight status limit the ability to generalise

Table 5. Inter-correlations between significant potential correlates of core food intake

(Pearson's $r$ correlation coefficients $\dagger$ )

\begin{tabular}{|c|c|c|c|}
\hline Correlations & $\begin{array}{l}\text { Maternal non-core } \\
\text { food intake in } 1999\end{array}$ & $\begin{array}{c}\text { Home non-core } \\
\text { food availability in } 2006\end{array}$ & $\begin{array}{l}\text { TV watching } \\
\text { in } 2006\end{array}$ \\
\hline Child's non-core food intake in 2006 & $0.25^{\star \star}$ & $0.34^{* *}$ & $0.33^{\star *}$ \\
\hline Maternal non-core food intake in 1999 & & -0.01 & $0.21^{\star \star}$ \\
\hline Home non-core food availability in 2006 & & & 0.06 \\
\hline
\end{tabular}




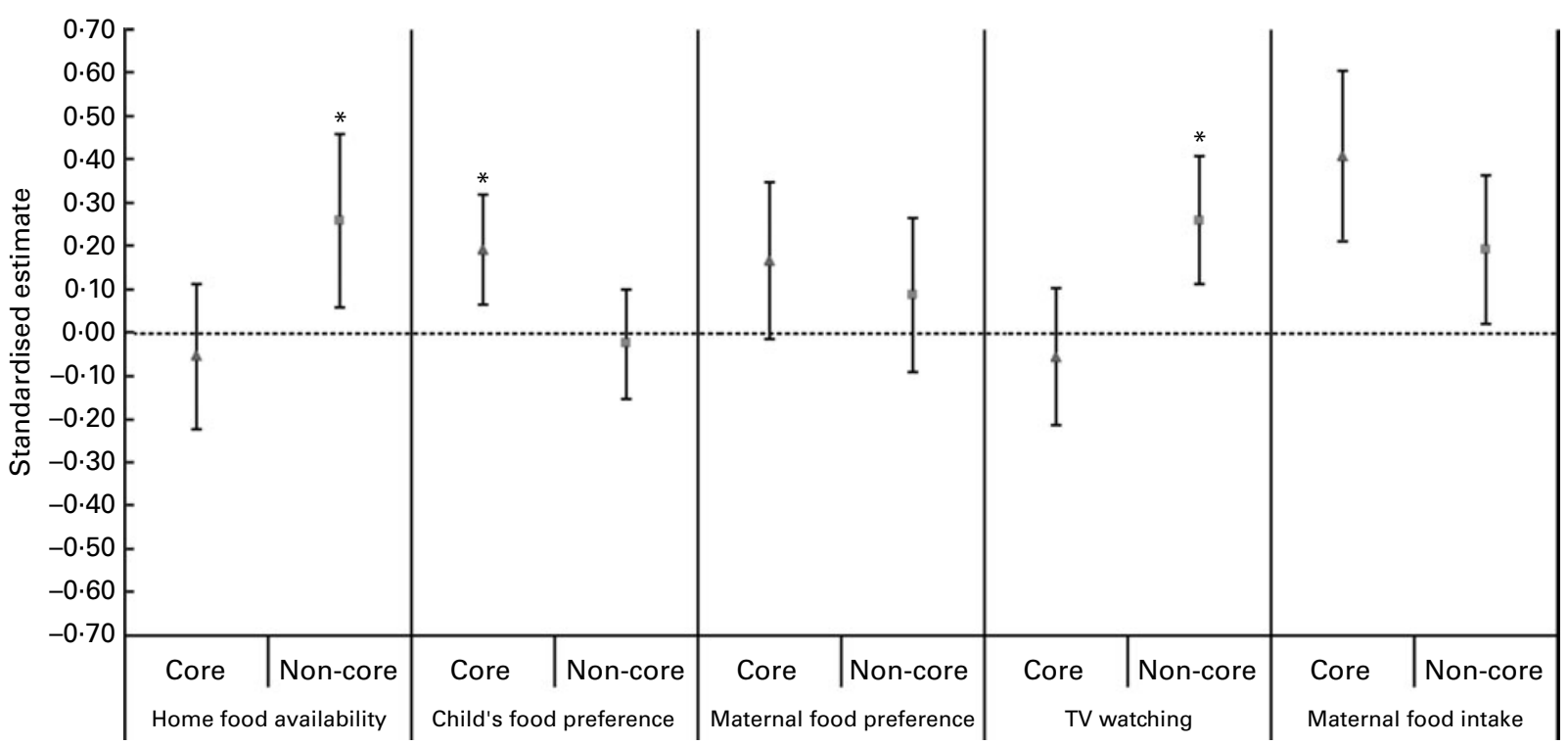

Fig. 1. Comparing the independent standardised correlates of core and non-core food intake in children $(n 219)$. Values are standardised estimates and $95 \% \mathrm{Cl}$ from a complex samples general linear model with either the child's core or non-core food intake as the outcome and including all potential correlates identified as significant in the fully adjusted models as independent variables. All variables were standardised with child's sex, child's age in 2006, child's BMI standard deviation score in 2006, maternal education and parental weight group at baseline as covariates. $Z$ test indicated if estimates were significantly different for core and non-core foods. * Standardised estimates were significantly different $(P<0.05)$. TV, television.

the results to a wider population of children; a largely cross-sectional design precludes interpretation of the direction of effects. Many measures were self-reported and may be biased by factors such as social desirability. Underreporting of children's TV watching by parent reports has been documented ${ }^{(48)}$ and could not be ruled out in the present analysis, which may mean that correlations between TV watching and food intake have been underestimated. The food intake measures lacked information on portion size, making it impossible to assess the quantity of food eaten on each occasion. Therefore, this analysis examined the variety of core and non-core foods consumed but could not investigate variation in the amounts eaten. Furthermore, parent food intake was measured 7 years before the measurement of food intake in children, which may not be representative of concurrent parental intakes. However, relative stability of diet in adulthood has been documented elsewhere ${ }^{(49)}$, suggesting that parents' intakes are likely to be ranked in a similar order over time. The strengths of the present analysis include the wide range of data available from both parents and their children on the potential correlates of food intake, which allowed a multivariable investigation of effects, and characterisation of the whole diet in terms of core and non-core foods rather than a narrow focus on just one or two food groups such as fruits and vegetables. Future studies with a larger sample size and adequate power to look at interactions might investigate the inter-relationships between the various correlates of food intake to create a more comprehensive model of food intake in children; pathways of action suggested by the attenuation effects observed in the present paper may be confirmed in this way.

\section{Conclusion}

Parental intake was associated with the child's intake for both core and non-core foods, but in addition, preferences were important for core foods but not for non-core foods, whereas availability and TV exposure were only important for non-core food intake. Longitudinal studies are needed to determine causality, but the present results suggest that interventions should have multiple targets at both the individual and family environment level and that different approaches may be appropriate to successfully change the balance of core and non-core foods in children's diets.

\section{Acknowledgements}

We gratefully acknowledge the ongoing contribution of the parents and children in the TEDS. The TEDS is supported by a programme grant (G0500079) from the UK Medical Research Council, and work on a subsample of the TEDS is supported in part by a grant from the Biotechnology and Biological Sciences Research Council (31/D19086) L. J., C. H. M. v. J. and J. W. are currently funded by a programme grant from Cancer Research UK (C1418/A7974). L. J. had the idea for the analysis, analysed the data and drafted the manuscript. J. W. designed the study, supervised the data collection, and advised on the idea, analysis and interpretation of the findings. C. H. M. v. J. advised on the idea, analysis and interpretation of the findings. All authors were responsible for critical revisions to the manuscript and approval of the final version. The authors have no conflicts of interest. 


\section{References}

1. US Department of Agricuture (USDA) \& US Department of Health and Human Services (USHHS) (2005) Dietary Guidelines for Americans 2005. http://www.health.gov/ dietaryguidelines/dga2005/document/pdf/DGA2005.pdf (accessed 24 May 2009).

2. Johnson L, Mander AP, Jones LR, et al. (2008) Energy-dense, low-fiber, high-fat dietary pattern is associated with increased fatness in childhood. Am J Clin Nutr 87, 846-854.

3. Chatzi L, Apostolaki G, Bibakis I, et al. (2007) Protective effect of fruits, vegetables and the Mediterranean diet on asthma and allergies among children in Crete. Thorax $\mathbf{6 2}$, 677-683.

4. Feinstein L, Sabates R, Sorhaindo A, et al. (2008) Dietary patterns related to attainment in school: the importance of early eating patterns. J Epidemiol Community Health 62, 734-739.

5. Department of Health (DOH) \& The Information Centre for health and social care (IC) (2008) Health Survey for England 2007: Latest trends 2008. http://www.ic.nhs.uk/ statistics-and-data-collections/health-and-lifestyles-relatedsurveys/health-survey-for-england/health-survey-for-england-2007-latest-trends-\%5Bns\%5D (published 16 December 2008).

6. Glynn L, Emmett P \& Rogers I (2005) Food and nutrient intakes of a population sample of 7 -year-old children in the south-west of England in 1999/2000 - what difference does gender make? J Hum Nutr Diet 18, 7-19, quiz 21-23.

7. McCarthy SN, Robson PJ, Livingstone MBE, et al. (2006) Associations between daily food intake and excess adiposity in Irish adults: towards the development of food-based dietary guidelines for reducing the prevalence of overweight and obesity. Int J Obes 30, 993-1002.

8. Bell AC, Kremer PJ, Magarey AM, et al. (2005) Contribution of 'noncore' foods and beverages to the energy intake and weight status of Australian children. Eur J Clin Nutr 59, 639-645.

9. Spurrier NJ, Magarey AA, Golley R, et al. (2008) Relationships between the home environment and physical activity and dietary patterns of preschool children: a cross-sectional study. Int J Behav Nutr Phys Act 5, 31.

10. Rangan AM, Randall D, Hector DJ, et al. (2008) Consumption of 'extra' foods by Australian children: types, quantities and contribution to energy and nutrient intakes. Eur J Clin Nutr 62, 356-364.

11. Webb KL, Lahti-Koski M, Rutishauser I, et al. (2006) Consumption of 'extra' foods (energy-dense, nutrient-poor) among children aged 16-24 months from western Sydney, Australia. Public Health Nutr 9, 1035-1044.

12. Patrick H \& Nicklas TA (2005) A review of family and social determinants of children's eating patterns and diet quality. $J$ Am Coll Nutr 24, 83-92.

13. van der Horst K, Oenema A, Ferreira I, et al. (2007) A systematic review of environmental correlates of obesityrelated dietary behaviors in youth. Health Educ Res 22, 203-226.

14. McClain A, Chappuis C, Nguyen-Rodriguez S, et al. (2009) Psychosocial correlates of eating behavior in children and adolescents: a review. Int J Behav Nutr Phys Act 6, 54 .

15. Campbell KJ, Crawford DA \& Ball K (2006) Family food environment and dietary behaviors likely to promote fatness in 5-6 year-old children. Int J Obes 30, 1272-1280.

16. Kourlaba G, Panagiotakos DB, Mihas K, et al. (2009) Dietary patterns in relation to socio-economic and lifestyle characteristics among Greek adolescents: a multivariate analysis. Public Health Nutr 12, 1366-1372.
17. Aranceta J, Perez-Rodrigo C, Ribas L, et al. (2003) Sociodemographic and lifestyle determinants of food patterns in Spanish children and adolescents: the enKid study. Eur J Clin Nutr 57, Suppl. 1, S40-S44.

18. Halford JC, Boyland EJ, Hughes GM, et al. (2008) Beyondbrand effect of television food advertisements on food choice in children: the effects of weight status. Public Health Nutr 11, 897-904.

19. Rosenkranz RR \& Dzewaltowski DA (2008) Model of the home food environment pertaining to childhood obesity. Nutr Rev 66, 123-140.

20. Halford JC, Gillespie J, Brown V, et al. (2004) Effect of television advertisements for foods on food consumption in children. Appetite 42, 221-225.

21. Jago R, Nicklas T, Yang SJ, et al. (2005) Physical activity and health enhancing dietary behaviors in young adults: Bogalusa Heart Study. Prev Med 41, 194-202.

22. Sanchez-Villegas A, Bes-Rastrollo M, Martinez-Gonzalez MA, et al. (2006) Adherence to a Mediterranean dietary pattern and weight gain in a follow-up study: the SUN cohort. Int J Obes 30, 350-358.

23. Park SY, Murphy SP, Wilkens LR, et al. (2005) Dietary patterns using the Food Guide Pyramid groups are associated with sociodemographic and lifestyle factors: the multiethnic cohort study. J Nutr 135, 843-849.

24. Craig LC, McNeill G, Macdiarmid JI, et al. (2010) Dietary patterns of school-age children in Scotland: association with socio-economic indicators, physical activity and obesity. BrJ Nutr 103, 319-334.

25. Jago R, Ness A, Emmett P, et al. (2009) Obesogenic diet and physical activity: independent or associated behaviours in adolescents? Public Health Nutr 3, 1-9.

26. Ventura AK \& Birch LL (2008) Does parenting affect children's eating and weight status? Int J Behav Nutr Phys Act 5, 15.

27. Wardle J (1995) Parental influences on children's diets. Proc Nutr Soc 54, 747-758.

28. Birch LL (1999) Development of food preferences. Annu Rev Nutr 19, 41-62.

29. Wardle J, Cooke LJ, Gibson EL, et al. (2003) Increasing children's acceptance of vegetables; a randomized trial of parent-led exposure. Appetite 40, 155-162.

30. Pearson N, Biddle SJH \& Gorely T (2009) Family correlates of fruit and vegetable consumption in children and adolescents: a systematic review. Public Health Nutr 12, 267-283.

31. Oliver BR \& Plomin R (2007) Twins' Early Development Study (TEDS): a multivariate, longitudinal genetic investigation of language, cognition and behavior problems from childhood through adolescence. Twin Res Hum Genet 10, 96-105.

32. Wardle J, Guthrie C, Sanderson S, et al. (2001) Food and activity preferences in children of lean and obese parents. Int J Obes 25, 971-977.

33. Hammond J, Nelson M, Chinn S, et al. (1993) Validation of a food frequency questionnaire for assessing dietary intake in a study of coronary heart disease risk factors in children. Eur J Clin Nutr 47, 242-250.

34. Bingham SA, Gill C, Welch A, et al. (1994) Comparison of dietary assessment methods in nutritional epidemiology: weighed records $v$. $24 \mathrm{~h}$ recalls, food-frequency questionnaires and estimated-diet records. Br J Nutr 72, 619-643.

35. Wardle J, Sanderson S, Leigh Gibson E, et al. (2001) Factor-analytic structure of food preferences in four-yearold children in the UK. Appetite 37, 217-223.

36. Nicklaus S, Boggio V, Chabanet C, et al. (2004) A prospective study of food preferences in childhood. Food Qual Prefer 15, 805-818

37. Birch LL, Fisher JO, Grimm-Thomas K, et al. (2001) Confirmatory factor analysis of the Child Feeding Questionnaire: 
a measure of parental attitudes, beliefs and practices about child feeding and obesity proneness. Appetite 36, 201-210.

38. Wardle J, Sanderson S, Guthrie CA, et al. (2002) Parental feeding style and the inter-generational transmission of obesity risk. Obesity 10, 453-462.

39. Reilly JJ, Penpraze V, Hislop J, et al. (2008) Objective measurement of physical activity and sedentary behaviour: review with new data. Arch Dis Child 93, 614-619.

40. Paternoster R, Brame R, Mazerolle P, et al. (1998) Using the correct statistical test for the equality of regression coefficients. Criminology 36, 859-866.

41. Clogg CC, Petkova E \& Haritou A (1995) Statistical methods for comparing regression coefficients between models. Am J Soc 100, 1261-1293.

42. Oliveria SA, Ellison RC, Moore LL, et al. (1992) Parent-child relationships in nutrient intake: the Framingham Children's Study. Am J Clin Nutr 56, 593-598.

43. Vauthier J-M, Lluch A, Lecomte E, et al. (1996) Family resemblance in energy and macronutrient intakes: the stanislas family study. Int J Epidemiol 25, 1030-1037.
44. Elfhag K, Tholin S \& Rasmussen F (2008) Consumption of fruit, vegetables, sweets and soft drinks are associated with psychological dimensions of eating behaviour in parents and their 12-year-old children. Public Health Nutr 23, $1-10$.

45. Blissett J, Meyer C \& Haycraft E (2006) Maternal and paternal controlling feeding practices with male and female children. Appetite 47, 212-219.

46. Wardle J \& Cooke L (2008) Genetic and environmental determinants of children's food preferences. BrJ Nutr 99, Suppl. 1, S15-S21.

47. Russell CG \& Worsley A (2007) Do children's food preferences align with dietary recommendations? Public Health Nutr 10, 1223-1233.

48. Rossiter JR \& Robertson TS (1975) Children's television viewing: an examination of parent-child consensus. Sociometry 38, 308-326.

49. Borland SE, Robinson SM, Crozier SR, et al. (2007) Stability of dietary patterns in young women over a 2 -year period. Eur J Clin Nutr 62, 119-126. 